\title{
Corrigendum
}

\section{TRAF2 inhibits TRAIL- and CD95L-induced apoptosis and necroptosis}

\author{
I Karl, M Jossberger-Werner, N Schmidt, S Horn, M Goebeler, M Leverkus, H Wajant and T Giner \\ Cell Death and Disease (2014) 5, e1556; doi:10.1038/cddis.2014.528; published online 4 December 2014
}

Correction to: Cell Death and Disease (2014) 5, e1444; doi:10.1038/cddis.2014.404; published online 9 October 2014

Since the publication of this paper it has been noted that there was an error in the abstract. The correct abstract is shown below.

The relevance of the adaptor protein TNF receptorassociated factor 2 (TRAF2) for signal transduction of the death receptor tumour necrosis factor receptor1 (TNFR1) is well-established. The role of TRAF2 for signalling by CD95 and the TNF-related apoptosis inducing ligand (TRAIL) DRs, however, is only poorly understood. Here, we observed that knockdown (KD) of TRAF2 sensitised keratinocytes for TRAIL- and CD95L-induced apoptosis. Interestingly, while cell death was fully blocked by the pan-caspase inhibitor benzyloxycarbonyl-Val-Ala-Asp(OMe)-fluoromethylketone (zVAD-fmk) in control cells, TRAF2-depleted keratinocytes were only partly rescued from TRAIL- and CD95L-induced cell death. In line with the idea that the only partially protective effect of zVAD-fmk on TRAIL- and CD95L-treated TRAF2depleted keratinocytes is due to the induction of necroptosis, combined treatment with zVAD-fmk and the receptor interacting protein 1 (RIP1) inhibitor necrostatin-1 fully rescued these cells. To better understand the impact of
TRAF2 levels on RIP1- and RIP3-dependent necroptosis and RIP3-independent apoptosis, we performed experiments in HeLa cells that lack endogenous RIP3 and HeLa cells stably transfected with RIP3. HeLa cells, in which necroptosis has no role, were markedly sensitised to TRAIL-induced caspasedependent apoptosis by TRAF2 KD. In RIP3-expressing HeLa transfectants, however, KD of TRAF2 also strongly sensitised for TRAIL-induced necroptosis. Noteworthy, priming of keratinocytes with soluble TWEAK, which depletes the cytosolic pool of TRAF2-containing protein complexes, resulted in strong sensitisation for TRAIL-induced necroptosis but had only a very limited effect on TRAIL-induced apoptosis. The necroptotic TRAIL response was not dependent on endogenously produced TNF and TNFR signalling, since blocking TNF by TNFR2-Fc or anti-TNFa had no effect on necroptosis induction. Taken together, we identified TRAF2 not only as a negative regulator of DR-induced apoptosis but in particular also as an antagonist of TRAIL- and CD95L-induced necroptosis.

The authors have also noticed that Figures 3-6 were incorrect. The figures have now been corrected in the original article. The corrected article appears online together with this corrigendum. The authors would like to apologise for any inconvenience caused. 